\title{
Regulating Intermolecular Chain Interaction of Biopolymer with Natural Polyol for Flexible, Optically Transparent and Thermally Conductive Hybrids
}

\author{
Marjan Alsadat Kashfipour, Nitin Mehra, Russell S. Dent and Jiahua Zhu*
}

Natural polymers like cellulose are rich in hydrophilic groups those are able to form intermolecular and intramolecular hydrogen bonding interactions. The regulation of such interactions can be critical for tuning material functionality. In this work, we designed a sustainable hybrid comprising of sodium carboxymethyl cellulose (SCMC) and xylitol, in which the molecular interaction is tuned for significantly enhanced thermal conductivity (TC). With optimized ratio of SCMC and xylitol, enhanced TC of up to 1.75 times of neat SCMC was achieved in addition to excellent flexibility and optical transparency. These enhancements are attributed to the formation of new H-bonds between SCMC and xylitol molecules resulting in formation of homogenously distributed thermal bridges throughout the polymer matrix. The intermolecular interaction in SCMC-xylitol composites elucidates some of the fundamental factors (e.g. H-bond intensity) responsible for promoted phonon transfer in polymeric materials, and at the same time sheds light on the capability of employing biopolymer-polyol based materials for thermal management applications.

Keywords: Biopolymer; Thermal conduction; Plasticizer; Cellulose; Xylitol

Received 8 March 2019, Accepted 12 April 2019

DOI: $10.30919 / \mathrm{es} 8 \mathrm{~d} 508$

\section{Introduction}

(indent) Thermal management is crucial in a variety of applications such as sensing, actuation and computation. The rapid trend of miniaturization over the last decade has elevated thermal management as one of the critical design problems. The overriding goal in such applications is to effectively utilize the available energy and minimize the risk of failure because of excessive heat in components of the systems. ${ }^{1-2}$ For thin flexible electronic devices such as electronic papers, ${ }^{3}$ flexible organic light-emitting $\operatorname{diodes}^{4.5}$ and foldable solar cells, ${ }^{6}$ combination of high thermal conductivity (TC), flexibility and optical transparency is required. Therefore, fabrication of materials exhibiting a good combination of these properties have attracted attentions.

Polymers are attractive platform for new functional materials because they are non-corrosive, flexible, electrically insulating and offer important phase change properties at an affordable cost. Despite these attractive properties, the impact of plastics on environment and ecosystem has aroused huge concern nowadays. To develop thermal management materials, traditional petroleum-based polymers are not natural fit since they suffer from intrinsically low TC. Therefore, it is of paramount importance to find green alternatives with proper approaches for their TC enhancement.

Heat conduction in insulating materials, including polymeric materials, is regulated by the propagation of phonons, which are

Intelligent Composites Laboratory, Department of Chemical and Biomolecular Engineering, The University of Akron, Akron, OH 44325 USA

*E-mail: jzhu1@uakron.edu discrete units of heat energy analogous to photons. ${ }^{7}$ Heat can be transferred efficiently across a temperature gradient when the scattering of phonon is minimized. ${ }^{8}$ Polymers usually show low TC because there is significant phonon scattering between the polymer chains. ${ }^{9-10}$ The high phonon scattering is due to random entanglement of polymer chains and absence of efficient thermal network for phonon transfer. ${ }^{11}$ Traditionally, TC enhancement of polymers can be achieved by incorporating thermally conductive fillers such as metallic, ${ }^{12-14}$ ceramic $^{15-17}$ or carbon based. $^{1820}$ When such fillers form a continuous network inside the matrix, efficient phonon propagation along the network would facilitate heat conduction. ${ }^{2}$ However, in terms of scale-up manufacturing, it is often challenging to inject a large amount of fillers to ensure the formation of such continuous networks because it increases the cost of material and also the difficulty in processing. ${ }^{21}$ In addition, the introduction of new polymer-filler interfaces would increase phonon scattering and adversely affect TC. ${ }^{22-24}$ Recently, Kim, et al. reported a significant enhancement of TC in miscible blend of poly (N-acryloyl piperidine) (PAP) and poly (acrylic acid) (PAA) without using any fillers. Interestingly, the formation of strong H-bonds between PAP and PAA resulted in the formation of continuous H-bond network inside the matrix and therefore significantly increased TC. It is claimed that such a network can be obtained by fine tuning the interchain interactions between the polymer chains. ${ }^{21}$ Using the same strategy, Mehra et al. enhanced the TC of poly (vinyl alcohol) (PVA) with small molecules including water $^{25}$ and short organic molecules. ${ }^{7,22}$ It has been demonstrated that incorporation of small molecules which can form $\mathrm{H}$ bonds with PVA facilitated the formation of a continuous thermal network inside the matrix followed by boosting the phonon transfer and TC.

Inspired by the aforementioned approach and considering the alternative biopolymers instead of petroleum-based polymers, we 
investigated the effects of interchain interactions between a biopolymeric host and a naturally occurring sugar alcohol on the thermal, mechanical and optical properties of the resulting hybrid film. The selected host is sodium carboxymethyl cellulose (SCMC) and the additive is xylitol. SCMC is an anionic linear polysaccharide, which is an esterified derivative of cellulose ${ }^{26}$ that is widely used in industries such as food, ${ }^{27}$ agriculture and forestry, ${ }^{28}$ textile and paper $^{29}$, drug and cosmetics, ${ }^{30}$ ceramics, paints and lacquers, adhesives etc. ${ }^{31}$ Its wide applicability is largely because of its high viscosity, ${ }^{26,32-33}$ low cost, nontoxic, non-allergenic, ${ }^{3436}$ biodegradability ${ }^{33}$ and its oil and lipid barrier properties. ${ }^{33,37}$ However, the presence of strong H-bonds in SCMC leads to its poor thermoplasticity, which can be improved by reducing the inter-molecular interactions. ${ }^{38}$ Plasticizers are often added to improve the processability and flexibility of such biopolymers. ${ }^{39}$ In this work, xylitol as a natural polyol was selected as plasticizer due to its rich hydroxyl groups ${ }^{40}$ and relatively high $\mathrm{TC}^{41}$ The insertion of such small plasticizers into SCMC could increase the distance between polymer chains and thus their mobility. ${ }^{42}$ Moreover, it is expected that new Hbonds can be formed between SCMC and xylitol and thus new thermally conductive networks inside the polymeric matrix. Here, the effect of xylitol concentration on the mechanical, optical and thermal properties of SCMC/xylitol hybrids was investigated by various characterization techniques. The complete green nature of such hybrids with desired multi-functionality offers a new perspective of utilizing sustainable products for advanced materials design and applications.

\section{Experimental}

\subsection{Materials and sample preparation}

(indent) High viscosity grade SCMC and xylitol were purchased from Sigma-Aldrich. These products were used as received. Deionized (DI) water (Millipore) with minimum resistivity of $18.2 \mathrm{M} \Omega$ was used as solvent. To prepare the films, $5.0 \mathrm{~g}$ of SCMC was gradually added to $150 \mathrm{~mL}$ DI water while stirring mechanically. After obtaining a transparent viscous solution, the required amount of xylitol was added to the solution and mixed for 30 minutes. Blends with different weight ratios of SCMC: xylitol (5: 0 (SX0), 5: 1 (SX1), 5: 2 (SX2), 5: 4 (SX4) and 5: 5 (SX5)) were prepared. The transparent solutions were poured into glass petri dishes and dried at $55{ }^{\circ} \mathrm{C}$ for 5 days to obtain freestanding films. These films were used for all the other measurements.

\subsection{Characterization methods}

(indent) Fourier-transform infrared spectroscopy (FT-IR) characterizations were carried out using Perkin Elmer ATR FT-IR. Mechanical tests were conducted using ADMET 500 universal testing machine (MTEST Quattro, USA). For tensile test, films were cut into rectangle shaped samples with dimension of $80 \times 5 \mathrm{~mm}$ and the average values of the thickness from five different points were used. Five tensile measurements were performed on each sample to minimize the experimental error. TC measurements were carried out using C-Therm TCi Thermal Conductivity Analyzer. Thermal behavior of the samples was characterized by Thermogravimetric Analyzer (TGA Q50, TA instrument). The transmittance characterization of films was carried out using UV-1800 Shimadzu spectrophotometer in the range of 200-800 $\mathrm{nm}$.

\section{Results and discussion}

(indent) SCMC is a cellulose derivative with sodium carboxymethyl $\left(\mathrm{CH}_{2} \mathrm{COONa}\right)$ groups. ${ }^{26}$ The large number of hydroxyl and carboxyl groups present in SCMC, as illustrated in Fig. 1, is responsible for its hydrophilic nature. Since SCMC and xylitol are both hydrophilic, they are soluble and miscible in water. Xylitol molecules can easily diffuse into the intermolecular space between SCMC chains. When the water is evaporated, the mixture results in a transparent film.

To distinguish the molecular interactions by using FT-IR, the wavelength shift of a certain functional group should be monitored. ${ }^{43,4}$ Since SCMC and xylitol are hydrophilic components with numerous hydroxyl groups, their $\mathrm{O}-\mathrm{H}$ band shifts are a reliable indicator for
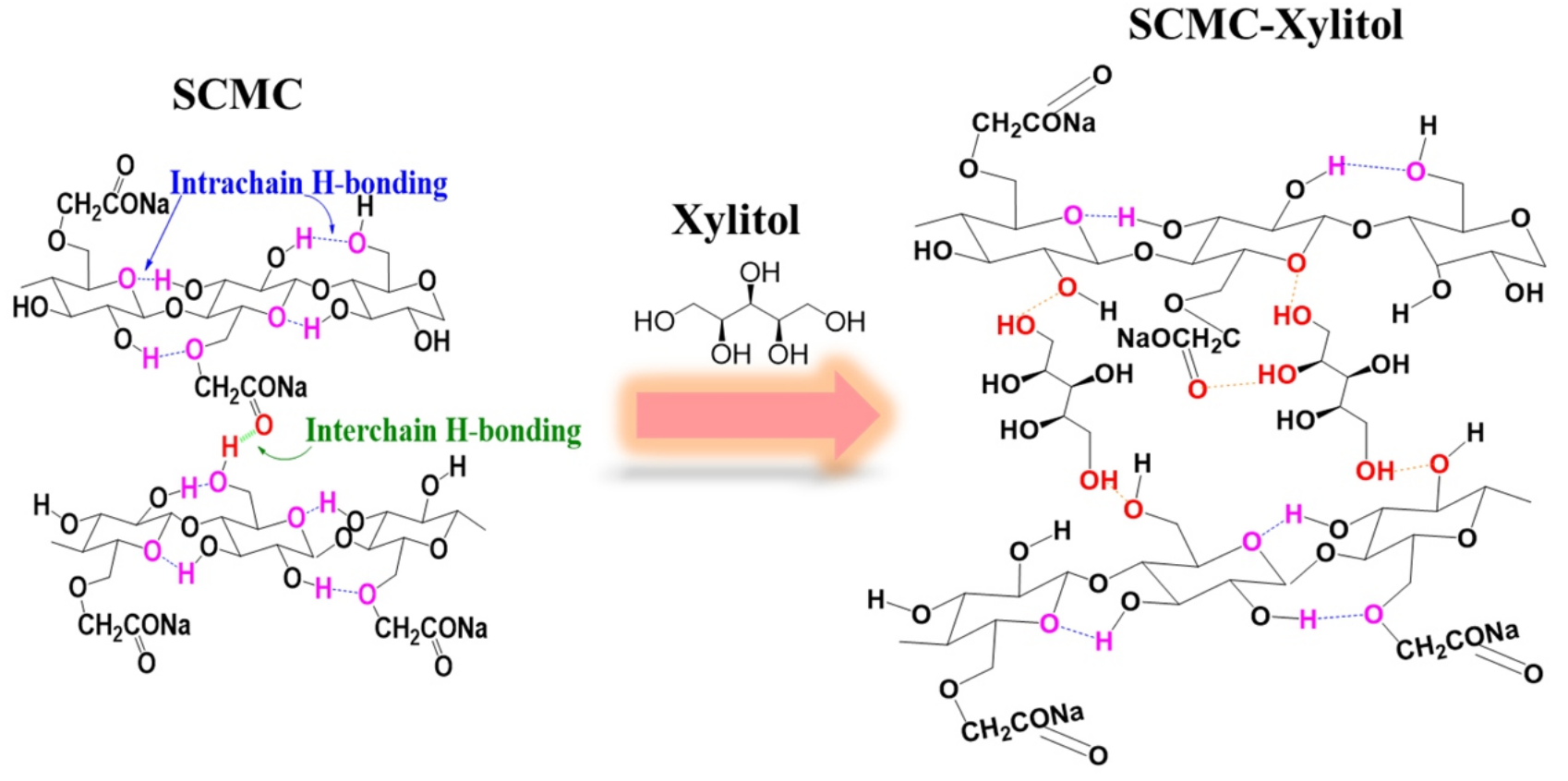

Fig. 1 The H-bonds involved in SCMC are illustrated; intrachain H-bonding are shown in blue and interchain H-bonding are shown in green. The Hbonds between the molecules of SCMC can be replaced by new H-bonds between xylitol and SCMC molecules. The shown configuration is only one of the possibilities in SCMC-xylitol system. 
monitoring the changes in interactions of the system. Fig. 2 shows the characteristic peaks associated with the main functional groups in SCMC. The peak at $2961.93 \mathrm{~cm}^{-1}$ is associated with the stretching of C$\mathrm{H}$ bonds while peaks at 1588.12 and $1413.13 \mathrm{~cm}^{-1}$ are ascribed to asymmetry and symmetry stretching of carboxylate groups, respectively. The band at $1024.51 \mathrm{~cm}^{-1}$ is assigned to the asymmetry stretching frequency of $\mathrm{C}-\mathrm{O}-\mathrm{C}$ groups. The strong and broad peak at $3318 \mathrm{~cm}^{-1}$ is associated with the stretching frequency of $\mathrm{O}-\mathrm{H}$ bonding as well as interchain and intrachain H-bonding. ${ }^{4546}$ These H-bonds are formed between SCMC molecules due to the presence of large number of hydroxyl and carboxyl groups. ${ }^{47}$ The $\mathrm{O}-\mathrm{H}$ bond stretching of xylitol appears at 3431,3364 and $3290 \mathrm{~cm}^{-1}$ wavelengths. ${ }^{48}$

Fig. 2(A) shows the FT-IR spectra of the neat SCMC and the SCMC-xylitol hybrid films with different contents of xylitol. The peaks representing the $\mathrm{O}-\mathrm{H}$ stretching of SCMC-xylitol system, shown in the highlighted wavelength range 3000-3550 $\mathrm{cm}^{-1}$ appears smooth for samples with xylitol up to $44.4 \mathrm{wt} \%$ (SX4). In contrast, for SX5 sample, there are multiple peaks, which appear at wavelength of 3425 , 3360 and $3293 \mathrm{~cm}^{-1}$. As discussed earlier, these peaks are the finger prints of xylitol. Therefore, it seems that this concentration of xylitol exceeds the compatibility limit of SCMC-xylitol system. ${ }^{49}$

Fig. 2(B) shows the O-H stretching peaks at higher magnification. The hydroxyl stretching peak shifted from 3335 to 3268,3276 and 3288 $\mathrm{cm}^{-1}$ for SX1, SX2 and SX4 confirming the enhanced strength of hydrogen bonding interactions. ${ }^{50}$ However, this is in contrast with the plasticizing role of xylitol. Plasticizing action of a plasticizer is accompanied with weakening of the attraction forces between the polymer chains leading to flexibility enhancement of the polymer. Thus, xylitol is acting as an antiplasticizer in these concentrations. Antiplasticizing effect or antiplasticization is the effect of low concentration of plasticizer in which they increase the stiffness of polymers. ${ }^{52}$ Previously, Chaudhary has demonstrated the antiplasticzing effect of polyols on the glass transition $\left(T_{g}\right)$ of starch films. It was found that incorporation of xylitol and glycerol in low concentration led to increased $T_{g}$. This phenomenon was explained by increased interactions followed by enhanced networking and molecular entanglements. ${ }^{53}$

Finally, the $\mathrm{OH}$ peak of the SX5 sample appeared with $\mathrm{OH}$ peaks of xylitol at higher wavelength than the corresponding wavelength in pure SCMC. The higher the wavelength, the weaker is the interaction. ${ }^{5455}$ This means that the strength of intermolecular hydrogen bonding of
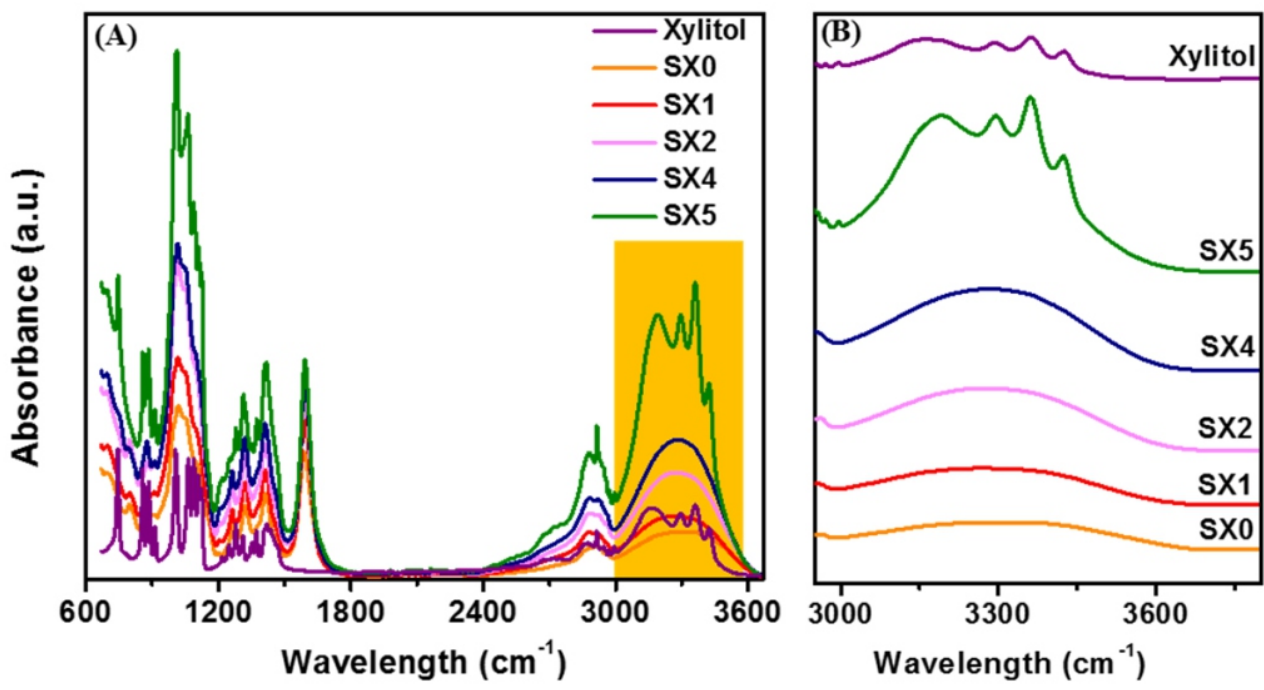

Fig. 2 (A) FT-IR spectra of xylitol and SCMC-xylitol films with different xylitol contents (SX0-SX5 respectively), (B) higher magnification of hydroxyl stretching peaks which is highlighted in Fig. 2(A).

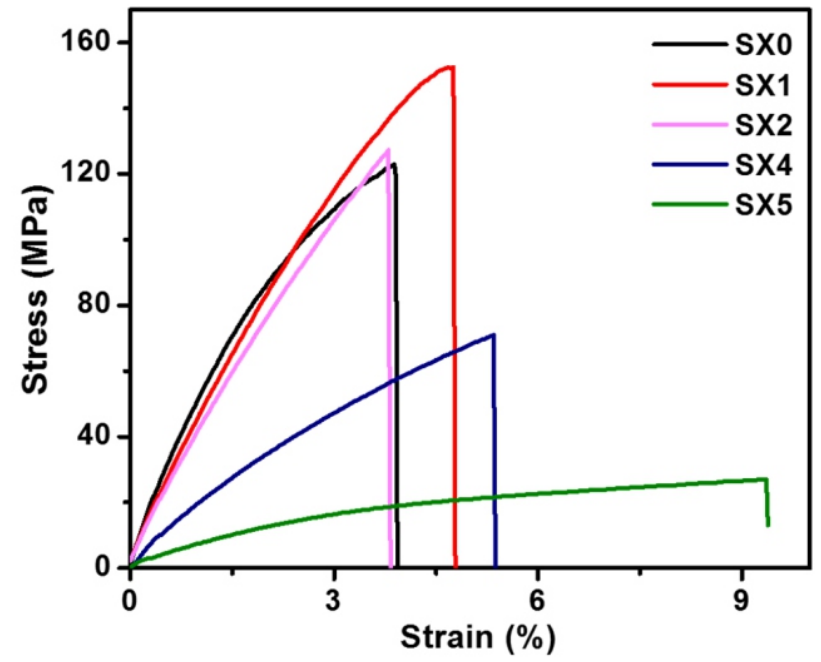

Fig. 3 Stress-strain curves of SCMC-xylitol films SX0- SX5 respectively. 
SCMC is decreased. Formation of H-bonds between SCMC and xylitol can affect the intra-molecular interaction of individual component ${ }^{42,53}$ and at the same time leads to the formation of thermal bridges, which facilitates phonon transfer across the polymer chain. ${ }^{11,21-22}$ The former increases the mobility and flexibility of the macromolecules ${ }^{37}$, while latter enhances the TC of the resulted blend films. ${ }^{11,21-22,25}$

SCMC, like most of other biopolymers, suffers from poor mechanical properties such as fragility and brittleness that limit its application in several industries. Fragility can be addressed by using plasticizers. The low molecular weight of plasticizers allows them to diffuse into the intermolecular spaces and reduce intermolecular forces. As a result, the free volume is increased resulting in enhanced molecular mobility, and hence making the biopolymer more flexible. When biodegradable plasticizers are used for biopolymers, the hybrids retain their biodegradability. Polyols (e.g. sorbitol, mannitol, xylitol, etc.) are hydrophilic natural plasticizers that can enhance the mechanical properties of the compatible hydrophilic biopolymers such as SCMC. ${ }^{37}$

Mechanical test was performed to evaluate the effect of xylitol content on mechanical properties of SCMC namely elongation at break (\%elongation), tensile strength (TS) and modulus of elasticity (ME). As shown in Fig. 3, introduction of xylitol up to $4.0 \mathrm{~g}$ into SCMC does not significantly affect the \%elongation of the hybrids, while for SX5, \%elongation is about 2 times of its value for pure SCMC. This phenomenon can be explained by the plasticizing effect of xylitol and weakened intermolecular bonds of SCMC with newly formed H-bonds between xylitol and SCMC. As a result, the polymer chains are less restricted leading to better flexibility. ${ }^{42,49}$

The maximum stress and ME of all samples is summarized in Fig. 4. It is observed that lower loading of xylitol into SCMC (SX1 and SX2) results in more rigid films which is reflected in both stress and
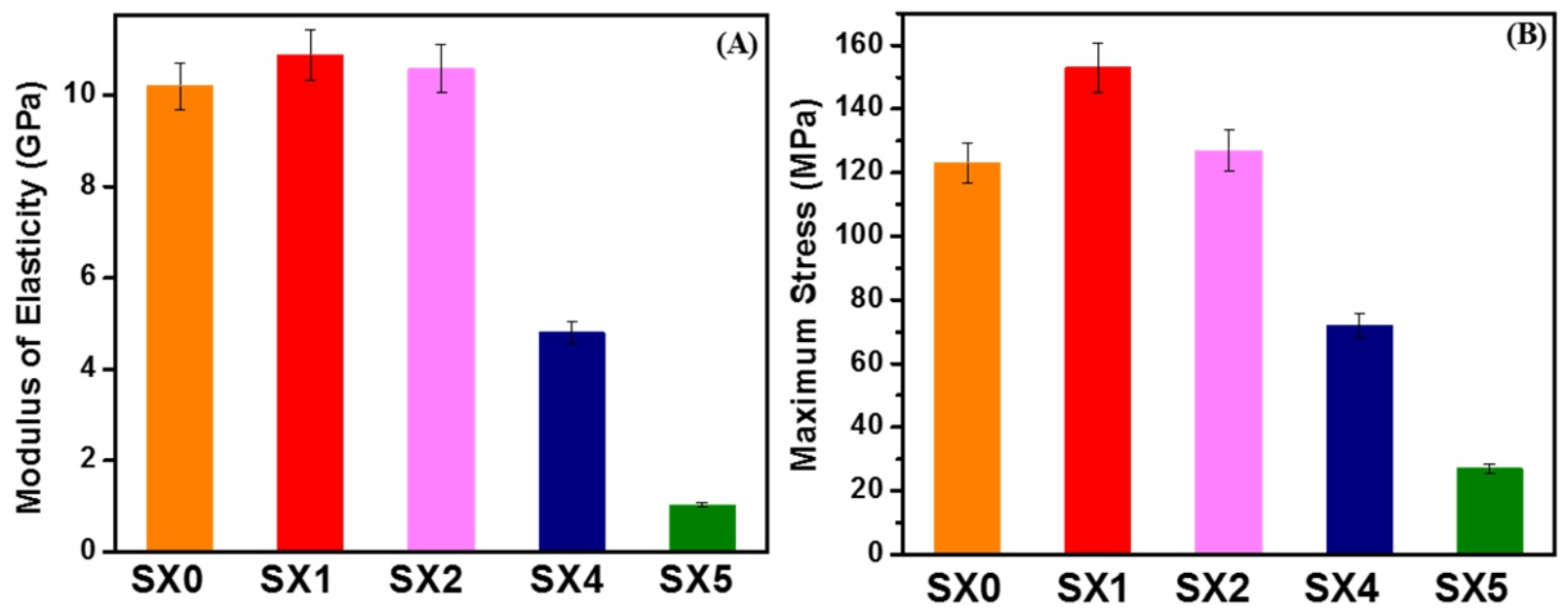

Fig. 4 (A) Summary of the effect of xylitol content on ME and (B) maximum stress (TS) of SCMC-xylitol films.
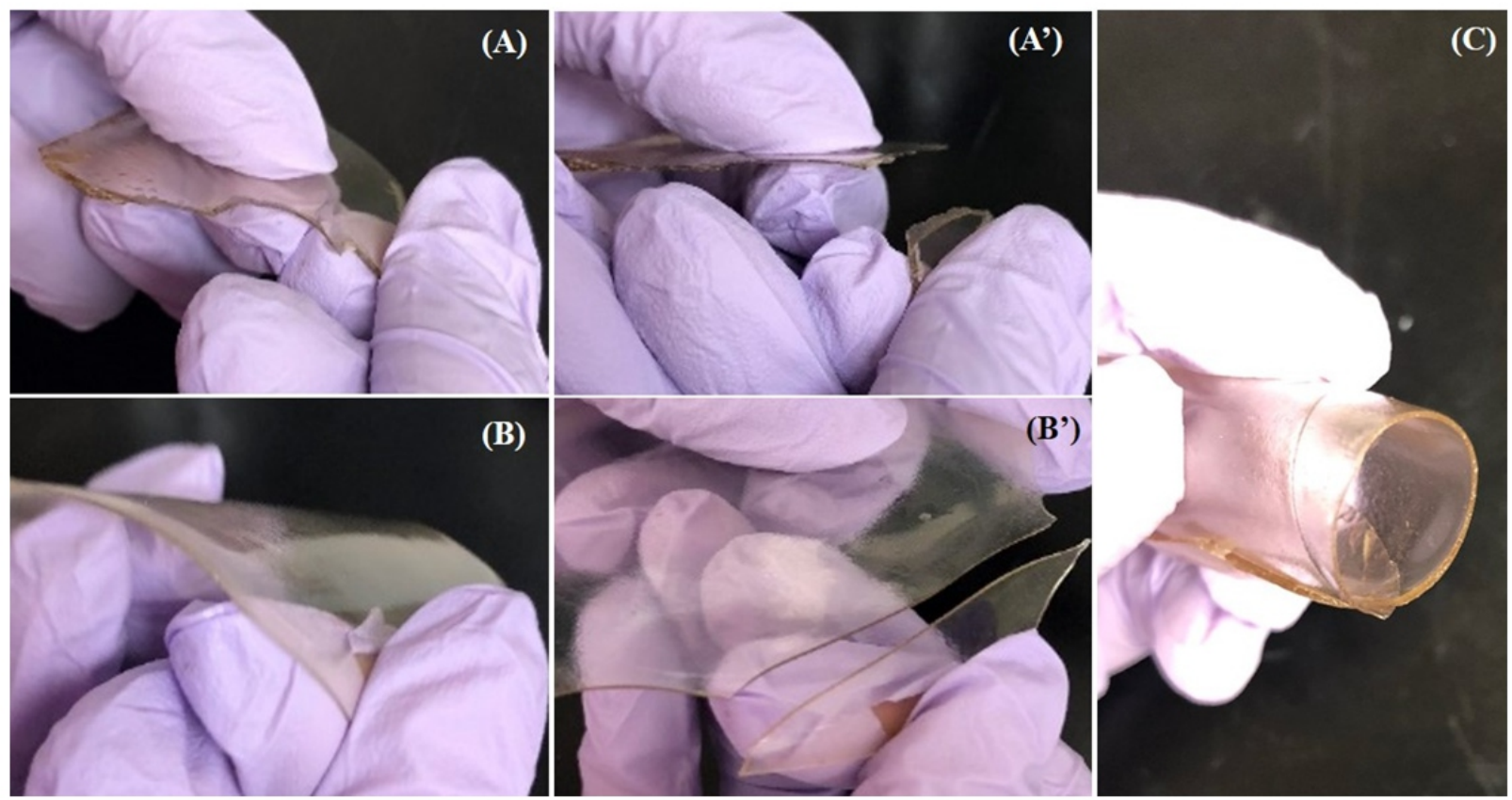

Fig. 5 (A) Before and (A') after applying force on SX0, SX1 and SX2 samples. These samples could not be bent and were broken immediately after bending. (B) Sample SX4 could be bent but broke when rolled (B'), and the SX5 sample was flexible enough to be rolled (C). 
ME. It is well-known that the mechanical properties are mainly affected by inter- and intramolecular interactions between the molecules. ${ }^{56}$ For SX1, TS improves from 123 to $153 \mathrm{MPa}$ and its ME increases from 10.21 to $10.88 \mathrm{GPa}$. Further addition of xylitol (SX2) decreased the TS to $127 \mathrm{MPa}$ and the ME to10.58 $\mathrm{GPa}$ which is still higher than strength and ME of the pure SCMC film. The increased strength and ME of SX1 and SX2 films can be explained by the antiplasticizing effect of xylitol. For SCMC-xylitol films with low content of xylitol, the hydroxyl groups of xylitol can increase the SCMC-xylitol interaction, networking and molecular entanglement that result in higher strength and ME. ${ }^{53,57}$ On the other hand, further increasing xylitol content in SX4 and SX5 samples, decreases both the strength and ME of the films which means xylitol is softening the films at these concentrations. Therefore, there is a transition zone for xylitol in the range of 28.5 to $44.4 \mathrm{wt} \%$ in which it switches from antiplasticizer to plasticizer for SCMC.

Fig. 5 shows the flexibility of the SCMC-xylitol films. Fig. 5(A) and Fig. 5(A') as the before and after applying bending, demonstrate the fragility of SX0, SX1 and SX2 films. These films could not bend and break immediately when bending force was applied. Similar phenomenon can be observed in SX4, Fig. 5(B\&B'). In contrast, SX5 can be rolled easily as illustrated in Fig. 5(C).

Fig. 6 shows the TC values of all the samples. The TC of neat $\mathrm{SCMC}$ is $0.43 \mathrm{~W} /(\mathrm{m} \cdot \mathrm{K})$ and it improved to $0.75 \mathrm{~W} /(\mathrm{m} \cdot \mathrm{K})(1.75$ times $)$ when the xylitol content was increased to $50 \mathrm{wt} \%$ (SX5). The formation of thermal conducting pathways between polymer chains that arise because of intermolecular interactions, i.e. H-bonding, among dissimilar molecules has been reported in the literatures. ${ }^{21-22,}{ }^{25,58}$ For instance, incorporation of water molecule in PVA matrix resulted in TC enhancement of over $200 \%$. TC improvement was based on the newly formed hydrogen bonding between the PVA chains and water molecules. ${ }^{25}$

Improving TC of polymeric systems with this approach requires a homogenous distribution of interchain interactions above the percolation threshold. ${ }^{21}$ Therefore, miscibility of the polymeric host with the additive is a key factor for enhancing TC. If the components are immiscible, the mixture will phase separate leading to agglomeration of polymer chains with increased phonon scattering, and thus lower TC. ${ }^{22}$ Here, due to hydrophilic nature of both SCMC and xylitol, H-bond can be formed uniformly within the mixture and thus improved TC was achieved.

As it was mentioned earlier, due to presence of hydroxyl and carboxyl groups in SCMC, abundant intra- and interchain hydrogen bonding is present in SCMC. These interactions lead to random selfassociation and rigidity of SCMC. The addition of xylitol disassociates such intrachain/interchain bonds (in SCMC) and forms new H-bonds with SCMC. The five hydroxyl groups in each xylitol molecule has great capability to interact with SCMC via H-bonds those are formed between the hydroxyl-hydroxyl groups or hydroxyl-carboxyl groups. As a result, a network of H-bonds through the matrix can be formed. Meanwhile, antiplasticizer effect of xylitol in low concentration increases the strength of present interactions followed by increased networking and molecular entanglements. Although plasticizer effect of xylitol in high concentration is accompanied with weakening of the interactions, but due to increased density of thermal bridges between the polymer chains a well-defined thermal network will be formed through the matrix. Therefore, the loading of thermal bridges through the matrix

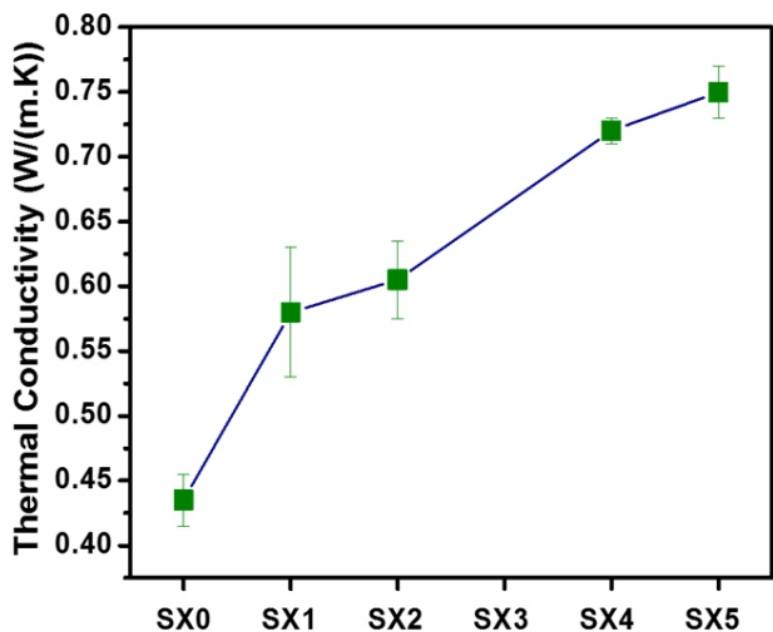

Fig. $6 \mathrm{TC}$ of SCMC-xylitol film increases with the content of xylitol in $5.0 \mathrm{~g}$ SCMC. Notice the improvement from $0.43 \mathrm{~W} /(\mathrm{m} \cdot \mathrm{K}$ ) (neat $\mathrm{SCMC})$ to $0.75 \mathrm{~W} /(\mathrm{m} \cdot \mathrm{K})$ with $50 \mathrm{wt} \%$ of xylitol.
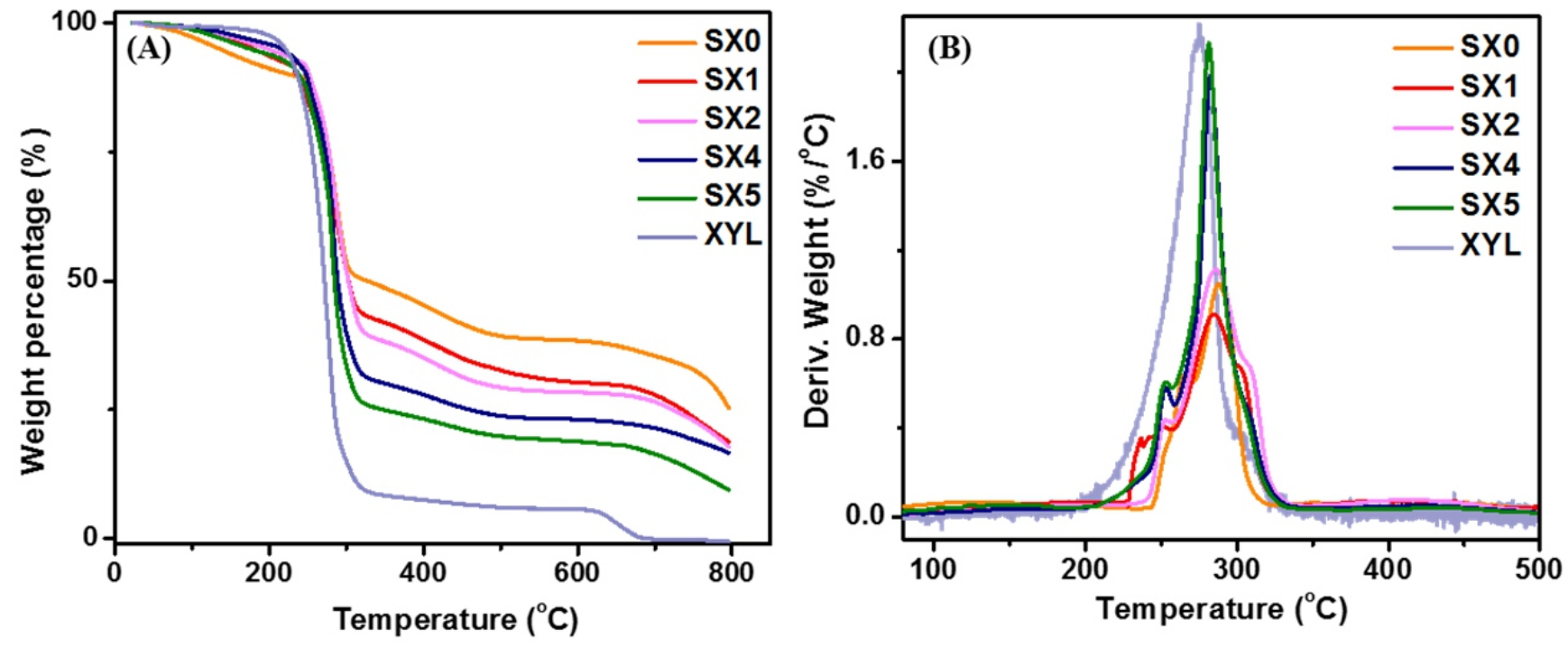

Fig. 7 (A) TGA and (B) Derivative TGA (DTGA) thermograms of SX0-SX5 samples and xylitol (Xyl). 
is a dominant factor over the strength of interactions, which results in enhancing TC up to $0.75 \mathrm{~W} /(\mathrm{m} \cdot \mathrm{K})$ with $50 \mathrm{wt} \%$ loading of xylitol.

Thermal stability of the hybrids was evaluated by TGA. Fig. 7(A) shows the thermal degradation of samples under nitrogen environment as a function of temperature. Decomposition of all the samples in a single step confirms the presence of a strong interaction between SCMC and xylitol. ${ }^{59-60}$ The initial decrease in weight of the samples that can be noticed in the Fig. 7(A) is due to the presence of moisture in the samples. In the temperature range of 250 to $323{ }^{\circ} \mathrm{C}$, the decrease in weight of SCMC can be attributed to decarboxylation. ${ }^{26,61}$ Finally in the temperature range of 373 to $511{ }^{\circ} \mathrm{C}$, the decrease in weight is because the remaining material in the film is converted into carbon residues. ${ }^{61}$ Since xylitol can be almost fully decomposed at $\sim 500{ }^{\circ} \mathrm{C}$, only SCMC residues remain at higher temperatures. The temperature, $\mathrm{T}_{\mathrm{dmax}}$, at which decrease in weight is maximum is illustrated in the DTGA curves presented in Fig. 7(B). The temperature at which the sample weight reduced by $50 \%$ was recorded as $\mathrm{T}_{50 \%}$. The weight of the samples at two specific temperatures, $300{ }^{\circ} \mathrm{C}$ and $500{ }^{\circ} \mathrm{C}$ were also recorded as shown in Table 1.

As shown in Table 1, presence of xylitol in the SCMC-xylitol mixtures resulted in reduced $\mathrm{T}_{50 \%}$ meaning that the degradation rate becomes higher. The weight percentage (\%) of the samples at 350 and $500{ }^{\circ} \mathrm{C}$ also confirmed that samples with higher content of xylitol lost more weight than other samples.

It is known that enhanced interaction between the polymer chains decreases the chains mobility and decomposition rate and enhances the thermal stability. ${ }^{62}$ However, it should be noted that the TC of reinforcing agents can also affect the thermal stability. Higher TC of additives can facilitate the diffusion of heat into the polymeric matrix. ${ }^{63-}$ ${ }^{64}$ As a result, in low content of xylitol the impact of enhanced heat diffusion is dominant over the enhanced interactions between the SCMC chains for the thermal stability. Similarly, increasing the content of xylitol is accompanied with enhancing the heat diffusion and also improving the chains mobility. However, the summarized results in Table 1 demonstrate that the maximum decomposition temperature of the SCMC-xylitol film was only reduced by $7^{\circ} \mathrm{C}$.

In spite of varying the xylitol content, all the films that were obtained in this study were optically transparent. No phase separation was observed even when the sample has $50 \mathrm{wt} \%$ of xylitol as illustrated in Fig. 8. In a polymeric system, phase separation can result in turbid slurries, ${ }^{22}$ exudated drops ${ }^{37}$ or in xylitol containing systems it can result in crystallization of xylitol. ${ }^{42}$ The absence of these phenomena in our system demonstrates sufficient compatibility between SCMC and xylitol even when the content of xylitol is high.

Fig. 8 shows that the increase of xylitol content can enhance the film transparency up to $\sim 93 \%$ in SX5 for the entire range of wavelength. In addition to the light barrier properties of the plasticizers $^{65}$, they can affect the polymer chain compaction and help to pass the light through polymeric films. ${ }^{66}$ In this work, the increased transparency of the SX5 film could be attributed to less compactness of SCMC chains.

\section{Conclusions}

(indent) Here, TC, optical transparency and flexibility of SCMC, which is a known biopolymer was successfully enhanced by engineering its interchain interactions. These enhanced properties were achieved with incorporation of xylitol, a natural sugar alcohol with 5 hydroxyl groups. As demonstrated, by increasing the content of xylitol TC increased from 0.43 to $0.75 \mathrm{~W} /(\mathrm{m} \cdot \mathrm{K})$, which represents a 1.75 times improvement compared to neat SCMC. TC enhancement at low concentration of xylitol was due to both strong H-bonding interactions and the newly formed thermal bridges between the polymer chains. At higher concentrations of xylitol, though H-bonding interactions were weakened, but resulted in increased number of thermal bridges. As a

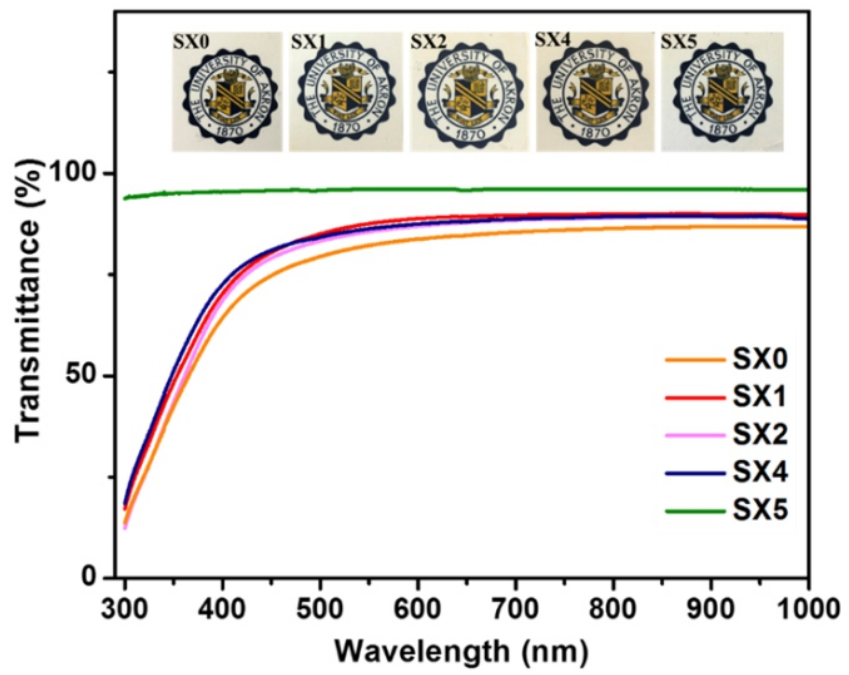

Fig. 8 UV-Vis and optical images of all the films. The above figures illustrate transparency of SX0, SX1, SX2, SX4 and SX5 films. The film containing $50 \mathrm{wt} \%$ of xylitol has the highest transmittance of $\sim 93 \%$.

Table $1 \mathrm{~T}_{\mathrm{dmax}}, \mathrm{T}_{50 \%}$ and weight loss (\%) of samples at 300 and $500{ }^{\circ} \mathrm{C}$ is shown. Increasing the content of xylitol resulted in faster thermal degradation and higher weight loss.

\begin{tabular}{ccccc}
\hline & & \multicolumn{2}{c}{ Weight loss (\%) } \\
Sample & $\mathrm{T}_{\mathrm{dmax}}\left({ }^{\circ} \mathrm{C}\right)$ & $\mathrm{T}_{50 \%}\left({ }^{\circ} \mathrm{C}\right)$ & $350{ }^{\circ} \mathrm{C}$ & $500{ }^{\circ} \mathrm{C}$ \\
\hline SX0 & 288.0 & 327 & 51.4 & 60.7 \\
SX1 & 285.0 & 303 & 58.1 & 67.4 \\
SX2 & 286.0 & 303 & 61.8 & 70.7 \\
SX4 & 283.0 & 289 & 69.9 & 76.2 \\
SX5 & 281.0 & 284 & 75.1 & 80.1 \\
Xylitol & 275.1 & 271.6 & 8.4 & 6.0 \\
\hline
\end{tabular}


result, a well-defined thermal network was formed through the matrix. In addition to the TC, xylitol affects the mechanical property of SCMC in two different ways. At low concentration, xylitol increases the brittleness of the SCMC films due to its antiplasticizing effect, reflecting strong $\mathrm{H}$-bonding interactions between the components while at higher concentrations, it acts as a plasticizer and enhances the flexibility of the films. Even though xylitol increased flexibility, the maximum decomposition temperature of the SCMC-xylitol film was only reduced by $7{ }^{\circ} \mathrm{C}$. In summary, incorporation of xylitol effectively enhances the TC and flexibility of SCMC with a negligible change in the maximum decomposition temperature. These materials provide a promising approach for the development of biodegradable materials for thermal management applications in flexible electronics.

\section{Notes}

The authors declare no competing financial interest.

\section{Acknowledgement}

Acknowledgement is made to the Donors of the American Chemical Society Petroleum Research Fund (\#55570-DNI10).

\section{References}

1. N. Burger, A. Laachachi, M. Ferriol, M. Lutz, V. Toniazzo and D. Ruch, Prog. Polym. Sci., 2016, 61, 1-28.

2. H. Chen, V. V. Ginzburg, J. Yang, Y. Yang, W. Liu, Y. Huang, L. Du and B. Chen, Prog. Polym. Sci., 2016, 59, 41-85.

3. Y. Chen, J. Au, P. Kazlas, A. Ritenour, H. Gates and M. McCreary, Nature, 2003, 423 (6936), 136.

4. Y. Okahisa, A. Yoshida, S. Miyaguchi and H. Yano, Compos. Sci. Technol., 2009, 69 (11-12), 1958-1961.

5. M. Nogi, H. Yano, Adv. Mater., 2008, 20 (10), 1849-1852.

6. M. Nogi, M. Karakawa, N. Komoda, H. Yagyu and T. T. Nge, Sci. Rep., 2015, 5, 17254

7. N. Mehra, L. Mu, T. Ji, X. Yang, J. Kong, J. Gu and J. Zhu, Appl. Mater. Today, 2018, 12, 92-130.

8. L. Mu, Y. Li, N. Mehra, T. Ji and J. Zhu, ACS appl. Mater. Inter., 2017, 9 (13), 12138-12145.

9. V. Singh, T. L. Bougher, A. Weathers, Y. Cai, K. Bi, M. T. Pettes, S. A. McMenamin, W. Lv, D. P. Resler and T. R. Gattuso, Nat. Nanotechnol. 2014, 9 (5), 384-390.

10. A. Henry, Аnпu. Rev. Heat Transfer, 2013, 17, 485-520.

11. N. Mehra, Y. Li and J. Zhu, J. Phys. Chem. C, 2018

12. I. Seshadri, G. L. Esquenazi, T. Borca-Tasciuc, P. Keblinski and G. Ramanath, Adv. Mater. Interfaces, 2015, 2 (15).

13. K. Pashayi, H. R. Fard, F. Lai, S. Iruvanti, J. Plawsky and T. Borca-Tasciuc, J. Appl. Phys., 2012, 111 (10), 104310.

14. Y. P. Mamunya, V. Davydenko, P. Pissis, E. Lebedev, Eur. Polym. J., 2002, 38 (9), 1887-1897.

15. T. Terao, Y. Bando, M. Mitome, C. Zhi, C. Tang and D. Golberg, J. Phys. Chem. C, 2009, 113 (31), 13605-13609.

16. T. Zhou, X. Wang, G. Mingyuan and X. Liu, Polymer, 2008, 49 (21), 46664672.

17. J. P. Hong, S. W. Yoon, T. Hwang, J. S. Oh, S. C. Hong, Y. Lee and J. D. Nam, Thermochim. Acta, 2012, 537, 70-75.

18. A. Yu, P. Ramesh, X. Sun, E. Bekyarova, M. E. Itkis and R. C. Haddon, $A d v$. Mater., 2008, 20 (24), 4740-4744.

19. M. Wang, Q. Kang and N. Pan, Appl. Therm. Eng., 2009, 29 (2-3), 418-421.

20. K. M. Shahil and A. A. Balandin, Nano lett., 2012, 12 (2), 861-867.

21. G. H. Kim, D. Lee, A. Shanker, L. Shao, M. S. Kwon, D. Gidley, J. Kim and K. P. Pipe, Nat. Mater., 2015, 14 (3), 295

22. N. Mehra, L. Mu and J. Zhu, Compos. Sci. Technol., 2017, 148, 97-105.

23. M. A. Kashfipour, N. Mehra and J. Zhu, Adv. Compos. Hybrid Mater., 2018, $1-25$

24. L. Mu, T. Ji, L. Chen, N. Mehra, Y. Shi and J. Zhu, ACS Appl. Mater. Int.,
2016, 8 (42), 29080-29087.

25. N. Mehra, L. Mu, T. Ji, Y. Li and J. Zhu, Compos. Sci. Technol., 2017, 151, 115-123.

26. D. Biswal and R. Singh, Carbohyd. Polym., 2004, 57 (4), 379-387.

27. V. Bifani, C. Ramírez, M. Ihl, M. Rubilar, A. García and N. Zaritzky, LWTFood Sci. Technol., 2007, 40 (8), 1473-1481.

28. H. Nie, M. Liu, F. Zhan and M. Guo, Carbohyd. Polym., 2004, 58 (2), 185189.

29. J. Chen, J.Wang, X. Zhang and Y. Jin, Mater. Chem. Phys., 2008, 108 (2-3), 421-424.

30. M. C. Adeyeye, A. C. Jain, M. K. Ghorab and W. J. Reilly, AAPS PharmSciTech, 2002, 3 (2), 16-25.

31. C. Hollabaugh, L. H. Burt and A. P. Walsh, Ind. Eng. Chem., 1945, 37 (10), 943-947.

32. J. H. Guo, G. Skinner, W. Harcum and P. Barnum, Pharmaceut. Sci. Technol. Today, 1998, 1 (6), 254-261.

33. L. Perioli, A. Dorigato, C. Pagano, M. Leoni and A. Pegoretti, Polymer Engineering \& Science.

34. K. A. A. Obeid, A. K. J. Al-Bermany and M. A. Habeeb, World Scientific News, 2015, 21, 12-23.

35. A. Gvrten, Acta Phys-Chim Sin., 2008, 24 (12), 2236-2242.

36. Y. Choi and J. Simonsen, J. Nanosci. Nanotechno., 2006, 6 (3), 633-639.

37. M. G. A. Vieira, M. A. Da Silva, L. O. Dos Santos and M. M. Beppu, Eur. Polym. J. 2011, 47 (3), 254-263.

38. X. Lin, Y. Li, Z. Chen, C. Zhang, X. Luo, X. Du and Y. Huang, Chem. Eng J., 2013, 215, 709-720.

39. J. S. Choi and W. H. Park, Polym. Test., 2004, 23 (4), 455-460.

40. X. Qiao, Z. Tang, and K. Sun, Carbohyd. Polym., 2011, 83 (2), 659-664.

41. E. P. Del Barrio, A. Godin, M. Duquesne, J. Daranlot, J. Jolly, W. Alshaer, T. Kouadio and A. Sommier, Sol. Energ. Mater. Sol. Cell., 2017, 159, 560-569.

42. R. A. Talja, H. Helén, Y. H. Roos and K. Jouppila, Carbohyd. Polym., 2007, 67 (3), 288-295.

43. Y. J. Yin, K. D. Yao, G. X. Cheng and J. B. Ma, Polym. Int., 1999, 48 (6), 429-432.

44. H. Liu, R. Adhikari, Q. Guo and B. Adhikari, J. Food Eng., 2013, 116 (2), 588-597.

45. R. Lin, A. Li, L. Lu and Y. Cao, Carbohyd. Polym., 2015, 118, 126-132.

46. M. Yu, J. Li and L. Wang, Chem. Eng. J., 2017, 310, 300-306.

47. W. Li, B. Sun and P. Wu, Carbohyd. Polym., 2009, 78 (3), 454-461.

48. F. Salaün, G. Bedek, E. Devaux, D. Dupont and L. Gengembre, J. Membrane Sci., 2011, 370 (1-2), 23-33.

49. D. Muscat, B. Adhikari, R. Adhikari and D. Chaudhary, J. Food Eng., 2012, 109 (2), 189-201.

50. A. Pawlak and M. Mucha, Thermochim. Acta, 2003, 396, 153-166.

51. V. V. Soman and D. S. Kelkar, Wiley Online Library, 2009, 152-161.

52. E. Snejdrova and M. Dittrich, In Recent advances in plasticizers; InTech: 2012.

53. D. S. Chaudhary, J. Appl. Polym. Sci., 2010, 118 (1), 486-495.

54. E. J. Moskala, S. E. Howe, P. C. Painter and M. M. Coleman, Macromolecules, 1984, 17 (9), 1671-1678.

55. M. Coleman, E. Moskala and R. Fti, Polymer, 1983, 24 (3), 251-257.

56. M. Shahbazi, S. J. Ahmadi, A. Seif and G. Rajabzadeh, Food Hydrocolloid., 2016, 61, 378-389.

57. H. G. Bader and D. Göritz, Part 2: water vapor sorption. Starch-Stärke, 1994, 46 (7), 249-252.

58. L. Mu, J. He, Y. Li,; T. Ji, N. Mehra, Y. Shi and J. Zhu, J. Phys. Chem. C, 2017, 121 (26), 14204-14212.

59. V. P. S. Nykänen, O. Härkönen, A. Nykänen, P. Hiekkataipale, J. Ruokolainen and O. Ikkala, Green Chem., 2014, 16 (9), 4339-4350.

60. J. F. Su, Z. Huang, X. Y. Yuan, X. Y. Wang and M. Li, Carbohyd. Polym., 2010, 79 (1), 145-153.

61. M. S. A. Rani, S. Rudhziah, A. Ahmad and N. S. Mohamed, Polymers, 2014, $6(9), 2371-2385$.

62. L. Mu, Y. Shi, H. Wang and J. Zhu, ACS Sust. Chem. Eng., 2016, 4 (3), 1840-1849.

63. C. Bao, L. Song, C. A. Wilkie, B. Yuan, Y. Guo, Y. Hu and X. Gong, J. Mater. Chem., 2012, 22 (32), 16399-16406.

64. C. Bao, L. Song, W. Xing, B. Yuan, C. A. Wilkie, J. Huang, Y. Guo and Y Hu, J. Mater. Chem., 2012, 22 (13), 6088-6096. 
65. Y. Zhang and J. Han, J. Food Sci., 2006, 71 (6), E253-E261.

66. R. Ortega-Toro, A. Jiménez, P. Talens and A. Chiralt, Carbohyd. Polym., 2014, 109, 155-165.
Publisher's Note Engineered Science Publisher remains neutral with regard to jurisdictional claims in published maps and institutional affiliations. 